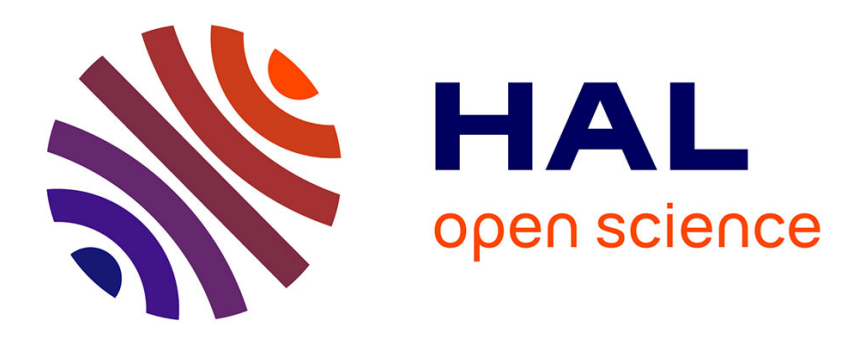

\title{
A numerical scheme for the computation of phase transition in compressible multiphase flows
}

\author{
Vincent Perrier
}

\section{To cite this version:}

Vincent Perrier. A numerical scheme for the computation of phase transition in compressible multiphase flows. Parallel CFD, Jun 2008, Lyon, France. inria-00341720

\section{HAL Id: inria-00341720 \\ https://hal.inria.fr/inria-00341720}

Submitted on 25 Nov 2008

HAL is a multi-disciplinary open access archive for the deposit and dissemination of scientific research documents, whether they are published or not. The documents may come from teaching and research institutions in France or abroad, or from public or private research centers.
L'archive ouverte pluridisciplinaire HAL, est destinée au dépôt et à la diffusion de documents scientifiques de niveau recherche, publiés ou non, émanant des établissements d'enseignement et de recherche français ou étrangers, des laboratoires publics ou privés. 


\title{
A numerical scheme for the computation of phase transition in compressible multiphase flows
}

\author{
Vincent Perrier ${ }^{1,2}$ \\ Université de Lyon 1 \\ Institut Camille Jordan and CDCSP \\ 43 Boulevard du 11 Novembre 1918 \\ 69622 Villeurbanne Cedex \\ perrier@math.univ-lyon1.fr
}

Summary. This paper is devoted to the computation of compressible multiphase flows involving phase transition. The compressible model is the system of Euler, without viscosity. For closing this model, an equation of state is required. In our context, the model of phase transition is included in the choice of the equation of state, via an entropy optimization of the mixture equation of state. Our aim is to simulate such a system, and for that, it is mandatory to understand well the Riemann problem with such an equation of state. We then propose a 2nd order numerical scheme, which is validated and proved to be accurate on one dimensional cases. Last, a $2 \mathrm{D}$ version of the code is proposed.

\section{Introduction}

In this paper, we are interested in the simulation of phase transition in compressible flows. The model is the inviscid compressible Euler system

$$
\left\{\begin{aligned}
\partial_{t} \rho+\operatorname{div}(\rho \mathbf{u}) & =0 \\
\partial_{t}(\rho \mathbf{u})+\operatorname{div}(\rho \mathbf{u} \otimes \mathbf{u}+P \mathbf{I}) & =0 \\
\partial_{t}(\rho E)+\operatorname{div}((\rho E+P) \mathbf{u}) & =0
\end{aligned}\right.
$$

where $\rho$ is the density, $\mathbf{u}$ the velocity, $P$ the pressure. $E$ is the total energy

$$
E=\frac{|\mathbf{u}|^{2}}{2}+\varepsilon
$$

where $\varepsilon$ is the specific internal energy. To close the system, an equation of state is necessary, that links the different thermodynamic parameters, for example $\varepsilon=\varepsilon(P, \rho)$.

The simulation of phase transition is difficult for (at least) two reasons 
1. We hope that the system is hyperbolic (this is true provided $\left(\frac{\partial P}{\partial \rho}\right)_{s}$ is positive). Numerical approximation of hyperbolic systems is difficult, because of the non uniqueness of the weak solution.

2. The very model of equation of state for modelling phase transition is still an open question. The most widely spread model is the van-der-Waals model of Bedjaoui and LeFloch [2002], Slemrod [1984]. In this case, system is not hyperbolic in the whole phase space, so that higher order terms are required for recovering the well-posedness of the Cauchy problem. In this paper, we are interested in a different model for which no regularization is needed.

This paper is organized as follows: in Section 1, we briefly record the model we use. In Section 2, we explain how to solve the Riemann problem with such a model of equation of state. Then in Section 3, we give a second order numerical scheme for simulating phase transition. In Section 4, we validate the scheme and give a two dimensions test.

\section{Thermodynamic model}

We denote by a subscript $l$ all that refers to the liquid, and by a subscript $g$ all that is linked to the gas. A subscript $i$ will be used when the equation holds for both of the phases. We suppose that each of the phase has its own equation of state. The total specific energy $\varepsilon$, specific entropy $s$, and specific volume $\tau(\tau=1 / \rho)$ are equal to

$$
\begin{gathered}
\varepsilon=y_{l} \varepsilon_{l}+\left(1-y_{l}\right) \varepsilon_{g} \\
s=y_{l} s_{l}+\left(1-y_{l}\right) s_{g} \\
\tau=y_{l} \tau_{l}+\left(1-y_{l}\right) \tau_{g}
\end{gathered}
$$

where $y_{l}$ is the mass fraction of the liquid. In order to reduce the number of unknowns, we choose the most stable mixture state, which is the one that optimizes the total entropy with fixed total specific volume and energy. As proved in Perrier [2008], the most stable state is

- either a pure liquid or a pure gas,

- or a mixture of both of the phases, with equality of pressure, temperature and energy.

To simplify, we suppose from now on that both of the phases are described by a perfect gas equation of state:

$$
\varepsilon_{i}\left(P_{i}, \tau_{i}\right)=\frac{P_{i} \tau_{i}}{\Gamma_{i}}
$$

where $\Gamma_{i}$ is the Grüneisen constant. As explained in Perrier [2008], this model is not able to account well for physics, but it nevertheless has the same mathematical characteristics as the realistic one. Moreover, all the computations can 
be led explicitly. All the details can be found in Perrier [2008]. If we denote by

$$
\alpha=\exp (1)\left(\frac{\Gamma_{2}^{\Gamma_{2}}}{\Gamma_{1}^{\Gamma_{1}}}\right)^{\frac{1}{\Gamma_{1}-\Gamma_{2}}},
$$

by $\tau_{i}=\Gamma_{i} / \alpha$, and if we suppose that $\Gamma_{l}<\Gamma_{g}$ then the situation is as follows.

- for $\tau<\tau_{l}$, the most stable state is the liquid,

- for $\tau>\tau_{g}$, the most stable state is the gas,

- for $\tau_{l}<\tau<\tau_{g}$, the most stable state is a mixture at thermodynamic equilibrium; it follows the equation of state $\varepsilon=P / \alpha$.

We note that the equation of state is continuous on $\tau=\tau_{i}$, but it has two differents derivative on the left and on the right. This means that the sound velocity is discontinuous. This will induce problems in solving the Riemann problem, and this is the issue of the next section.

\section{Solution of the Riemann problem}

Solving the Riemann problem is mandatory for building a numerical scheme based on a Godunov' method. The solution is well known when the equation of state has a continuous derivative, and when the isentropes are convex, see Godlewski and Raviart [1996]. Solving the Riemann problem for the Euler equations relies on computing the simple waves (see Godlewski and Raviart [1996]) for the waves $u \pm c$, and then intersecting them in the $(P, u)$ plane. Note that the computation of the velocity never deals with any problem, it is computed with the Riemann invariants or the Rankine-Hugoniot relations depending on the regularity of the wave. That is why we concentrate on the computation of the thermodynamic parameters along the wave curves.

As we said in the previous section, the derivatives of the equation of state are not continuous along $\tau=\tau_{i}$. Problems might occur when the wave curves cross $\tau=\tau_{i}$, which will be called in the following "phase transition". We then have to use the Liu criterion (see Liu [1975]) and other references (Wendroff [1972]) to build the wave curves. In the following, we detail how to compute them, depending on the thermodynamic state of the initial point.

\subsection{The initial point is a gas}

If a gaseous state undergoes an undercompressive wave, it begins by an isentrope, which means that $\tau$ increases. Therefore, it never meets any curve $\tau=\tau_{i}$, so that no phase transition occurs.

If a gaseous state undergoes a compressive wave, it begins by a shock, so that the line $\tau=\tau_{g}$ might be crossed. This is typically the situation described in Liu [1975]: the shock may be split into two shocks: a first one that leads to a saturated liquid (i.e. with $\tau=\tau_{l}$ ), followed by another shock. 


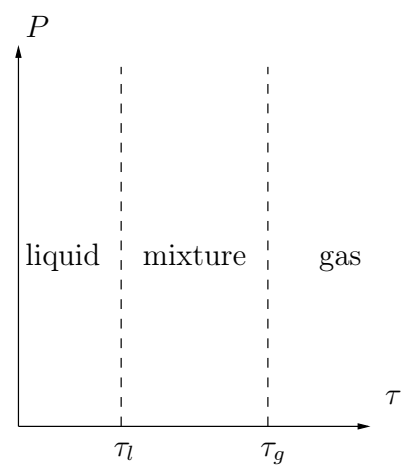

(a)

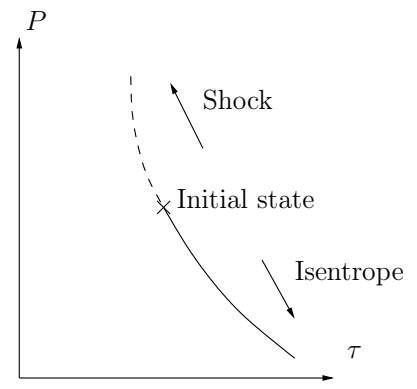

(b)

Fig. 1. (a): The thermodynamic plane is divided into three zones in which either a pure phase (liquid or gas) is stable, or a mixture is stable. On the lines $\tau=\tau_{i}$, the equation of state is continuous, but cannot be differentiated. (b): A wave curve in the thermodynamic plane $(\tau, P)$ is a decreasing curve. Under the initial state, the wave curve is an isentrope, whereas above, the wave curve is a shock.

\subsection{The initial point is a mixture}

If a mixture point undergoes a rarefaction wave, it may cross the line $\tau=\tau_{g}$. On this point, the characteristics of the left and right states are crossing, so that the wave curve cannot be composed only of an isentrope. Following Wendroff [1972], the rarefaction wave is a composite wave, which can be composed of

- a mixture isentrope,

- or a mixture isentrope, followed by an undercompressive discontinuity

- or a mixture isentrope, followed by an undercompressive discontinuity, followed by a gaseous isentrope,

- or an undercompressive discontinuity

- or an undercompressive discontinuity, followed by a gaseous isentrope.

If a mixture point undergoes a shock, the wave curve may cross the line $\tau=\tau_{l}$. Nevertheless, as the Hugoniot curve remains convex, the shock cannot be split (see Liu [1975]).

\subsection{The initial point is a liquid}

If a liquid point undergoes a shock, $\tau$ decreases, so that it does not cross any curve $\tau=\tau_{i}$. Therefore, no phase transition occurs.

If a liquid point undergoes a rarefaction wave, it may cross the line $\tau=\tau_{l}$. On this point, characteristics do not cross, so that the wave curve can be continued by a mixture rarefaction wave, which may be a composite wave, as seen in the previous subsection. 


\section{Numerical scheme}

\subsection{Numerical scheme}

Simulation of multiphase flows is a difficult task; the most advanced algorithms rely on the level set method, see Osher and Fedkiw [2003]. Nevertheless, this algorithm is not conservative, and therefore is not accurate for capturing shocks.

Another way to simulate multiphase flows if the Volume Of Fluid (VOF) method. It nevertheless deals with many geometrical problems, and is also not very developed in the compressible framework.

Moreover, these two algorithms are well suited for the simulation of material interfaces, in which the interface moves at the velocity $\mathbf{u}$, whereas in the phase transition context, they move at a sonic or subsonic velocity.

The numerical scheme we use is based on Abgrall and Saurel [2003], which was extended in Le Métayer et al. [2005] for taking into account phase transition waves. Nevertheless, in this last reference, the way to deal with mixture was left unclear. One of the problems in simulation of multiphase flows is that the strong disparities in the nonlinearity of the equation of state induces pressure oscillations, see Abgrall [1996]. In the phase transition context, the nonlinearity of the equation of state strongly changes from one to the other phase, but also from one phase to the mixture zone. That is why we use a three phase algorithm, for which the mixture at thermodynamic equilibrium is considered as a third phase.

\subsection{Second order extension}

The second order accuracy is achieved by a MUSCL-Hancock strategy: first, the variables are interpolated and then limited in each cell. This limitation is done with a Van Albada limiter, and also takes into account the thermodynamic stability of the states. More precisely,

- either the color function $\alpha$ is such that $0<\alpha<1$, and then the color function is interpolated, the phase thermodynamic parameters and velocity are not interpolated.

- or the color function $\alpha$ is equal to 0 or 1 , and the phase thermodynamic parameters and velocity are interpolated.

Then a time predictor-corrector scheme is used, with a special integration formula for dealing with nonconservative terms.

\section{Numerical results}

All the computations are made with $\Gamma_{l}=0.9$ and $\Gamma_{g}=0.2$. With this choice of Grüneisen coefficients, the limit of saturation of the phases are equal to $\rho_{l}=0.566$ and $\rho_{g}=2.544$. We first validate the code with Riemann problems in dimension 1 . We then give a 2 dimensional test. 


\subsection{Validation test 1: split rarefaction wave}

In the first test, the left state is composed of a mixture at thermodynamic equilibrium. Its density is equal to 1 , and its pressure is equal to $5 \times 10^{5} \mathrm{~Pa}$. The right state is composed of a gas, with density 0.1 and pressure $10^{5} \mathrm{~Pa}$. In the two sides, the velocity is equal to 0. Results obtained are shown on Figure 2 , and perfectly match with the analytical solution. The pressure ratio is such that it will induce a rarefaction wave in the mixture zone, which will be split because of the phase transition.
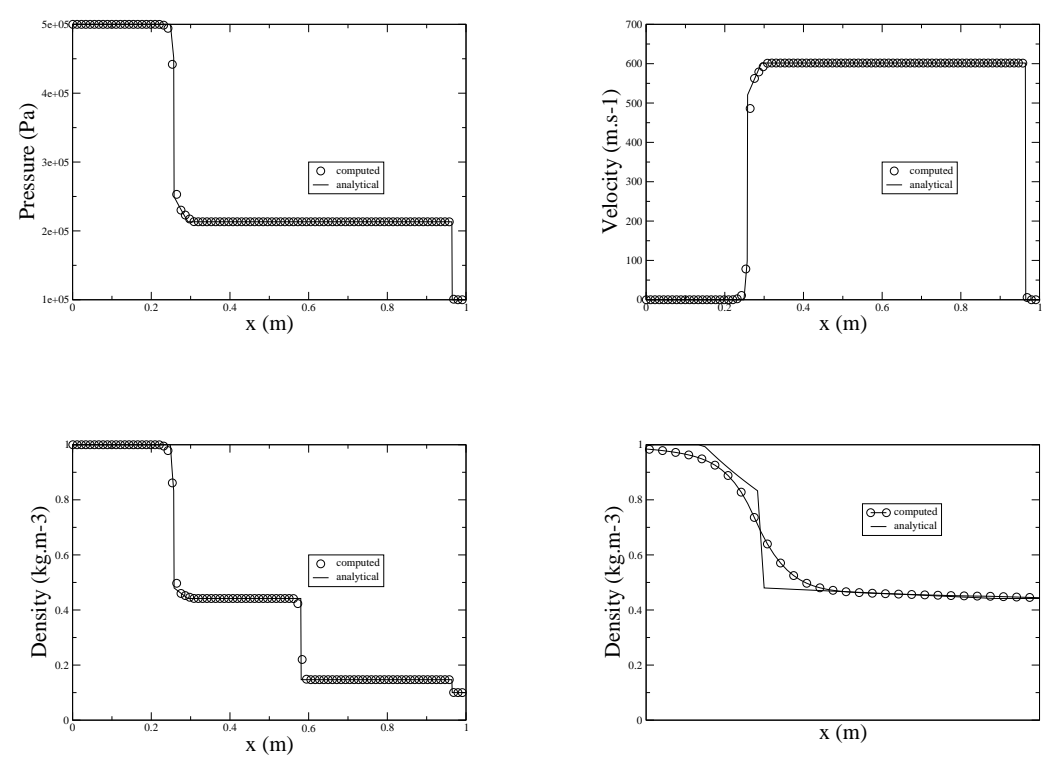

Fig. 2. Comparison of the numerical solution obtained and the analytical one for a split rarefaction wave for density, pressure and velocity. The last figure is a zoom on the split rarefaction wave.

\subsection{Validation test 2: Liu solution of a split shock}

In this test, the left state is a liquid, with velocity $100 \mathrm{~m} . \mathrm{s}^{-1}$, and the right state is a gas, with velocity $-100 \mathrm{~m} . \mathrm{s}^{-1}$. This induces a liquefaction shock, that is split, see Liu [1975]. Results and comment are on Figure 3.

\subsection{2 dimensional test}

In this test, we consider a metastable phase transition. For details on the modelling and the way to solve the Riemann problem, see Perrier [2008]. Results and comments are shown on Figure 4. 

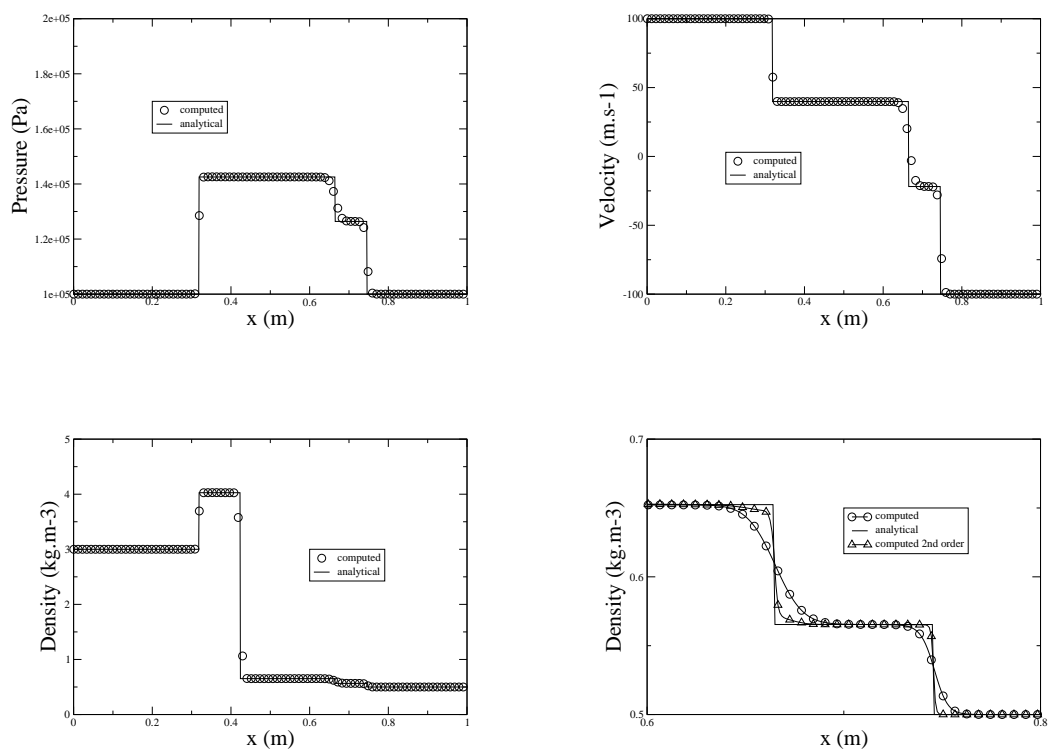

Fig. 3. Comparison of the numerical solution obtained and the analytical one for a shock splitting, for density, pressure and velocity. The last figure is a zoom on the split shock, in which we compare the first and second order computed solutions.

\section{References}

Rémi Abgrall. How to prevent pressure oscillations in multicomponent flow calculations: a quasi-conservative approach. J. Comput. Phys., 125(1):150160, 1996. ISSN 0021-9991.

Rémi Abgrall and Richard Saurel. Discrete equations for physical and numerical compressible multiphase mixtures. J. Comput. Phys., 186(2):361-396, 2003. ISSN 0021-9991.

Nabil Bedjaoui and Philippe G. LeFloch. Diffusive-dispersive traveling waves and kinetic relations. I. Nonconvex hyperbolic conservation laws. J. Differential Equations, 178(2):574-607, 2002. ISSN 0022-0396.

Edwige Godlewski and Pierre-Arnaud Raviart. Numerical approximation of hyperbolic systems of conservation laws, volume 118 of Applied Mathematical Sciences. Springer-Verlag, New York, 1996. ISBN 0-387-94529-6.

Olivier Le Métayer, Jacques Massoni, and Richard Saurel. Modelling evaporation fronts with reactive Riemann solvers. J. Comput. Phys., 205(2): 567-610, 2005. ISSN 0021-9991.

Tai Ping Liu. The Riemann problem for general systems of conservation laws. J. Differential Equations, 18:218-234, 1975. ISSN 0022-0396. 


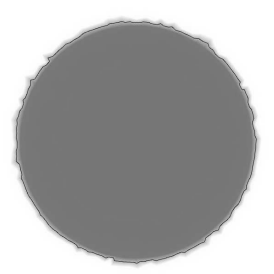

(a)

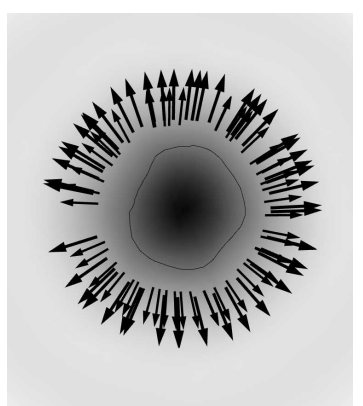

(c)

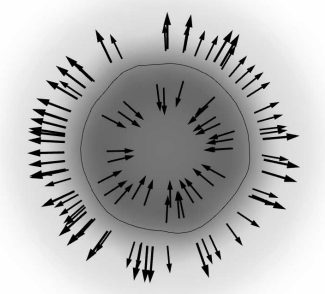

(b)

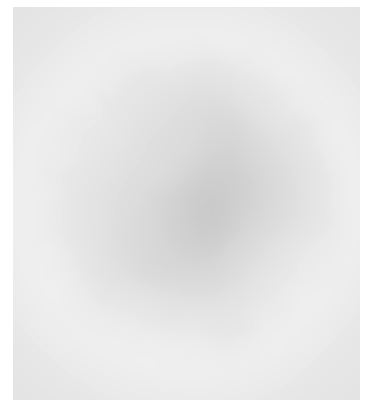

$(d)$

Fig. 4. On this four figures, the density is in grey-scale: the higher is the darker; the velocity is shown with arrows, and the bubble is represented with an iso of the volume fraction. At the beginning, a bubble of liquid is inside a gas $(a)$. The liquid is metastable, which induces a phase transition front, and sonic waves: a shock is emitted inside $(b)$, then focus in the center of the bubble $(c)$. Last, the bubble disappears $(d)$, and behaves as a single phase problem which goes at rest.

Stanley Osher and Ronald Fedkiw. Level set methods and dynamic implicit surfaces, volume 153 of Applied Mathematical Sciences. Springer-Verlag, New York, 2003. ISBN 0-387-95482-1.

Vincent Perrier. The Chapman-Jouguet closure for the Riemann problem with vaporization. SIAM J. Appl. Math., 68(5):1333-1359, 2008. ISSN 0036-1399.

Marshall Slemrod. Dynamic phase transitions in a van der Waals fluid. $J$. Differential Equations, 52(1):1-23, 1984. ISSN 0022-0396.

Burton Wendroff. The Riemann problem for materials with nonconvex equations of state. II. General flow. J. Math. Anal. Appl., 38:640-658, 1972. 